\title{
Precision improved spot centroid-locating algorithm based on profile regularization
}

\author{
Feng QIAN $^{1, a}$, Xiao-Pei ZHANG ${ }^{2, b}$ \\ ${ }^{1}$ University of Chinese Academy of Sciences, Beijing, 100049, China \\ ${ }^{2}$ Henry Samueli School of Engineering and Applied Science, University of California, Los Angeles, \\ 405 Hilgard Avenue, Los Angeles, CA 90095, USA \\ aEmail: zilgard@126.com, 'Email: zxpmirror1994@gmail.com
}

Keywords: centroid detection; profile regularization; center of weight

\begin{abstract}
The spot centroid localization is one of the key problems in various fields of scientific research and engineering applications. This paper proposed a novel algorithm based on profile regularization of the detecting spots. The algorithm first roughly eliminates the background and random noise errors by an a priori statistically estimated threshold; then determines the profile edge of the spot region by using morphological filtering technique; last calculates the centroid position with a center of weight algorithm. The field experiments were performed to validate the presented algorithm. The experimental results have demonstrated the superiorities of the proposed centroid detection algorithm on precision, stability and repeatability. This study is of value in improving the precision of center location algorithms.
\end{abstract}

\section{Introduction}

Centroid detection of spot images acts as a key role in measuring wave-front distortion by Shack-Hartmann wave-front sensor for adaptive optics[1][2], optical testing[3][4] and many other area such as pattern recognition for point object[5], freeform surface measurement[6], lithography system[7], etc. The measuring precision of the centroid for each spot directly affects the accuracy of measurement or assessment in such cases. The conventional centroid detection algorithm is based on the gray level of the spot image[8]. However, when there is noise in the spot image and the noise is not negligible compared to the signals, the detection of an accurate center position becomes difficult by solely using the conventional algorithm. Considering that the background noise may have influence to detect the center position, the modified center of weight algorithms have been developed. The modified versions take power of the gray level of the spot image intensity[9] instead of the gray level itself as the weighting factor. The general method to eliminate the background noise is threshold method[10] and windowing algorithm[11]. In the threshold method, a threshold is used to determine the boundary between spot signal and noise. In the windowing algorithm, the size of a detection window is changed to reduce the influence of noises. Image filtering technique is also introduced to remove the noise, such as smoothing filter, high-frequency removal filter, etc.

This study proposed a novel algorithm of locating spot centroids based on profile regularization. The algorithm estimates the profile edge of a pot more precisely based on the intrinsic spatial correlation of the spot, rather than an optimal estimated threshold, which is usually implied in segmentation algorithms. A center of weight algorithm is applied on the regularized spot region for centroid location. In the experiments section, the performance of the presented algorithm and its comparison with the reference method has been discussed. The conclusion is in the last section.

\section{Principles of Centroid Detection}

\section{Center of Weight algorithms.}

The conventional center of weight algorithm and its modified versions can be uniformly expressed as 


$$
\mathrm{x}_{c}=\frac{\sum_{i, j} I_{i j}^{\alpha} \cdot X_{i j}}{\sum_{i, j} I_{i j}^{\alpha}}, \quad \mathrm{y}_{c}=\frac{\sum_{i, j} I_{i j}^{\alpha} \cdot \mathrm{y}_{i j}}{\sum_{i, j} I_{i j}^{\alpha}},
$$

where $I_{i j}$ is the intensity of the pixel of $i$-th column and $j$-th row, $\alpha$ is the power of the intensity, $x_{i j}$ and $\mathrm{y}_{i j}$ are the position coordinates of the pixels in the calculated region, $\mathrm{x}_{c}$ and $\mathrm{y}_{c}$ are the detected center positions. When $\alpha$ equals 1 , Eq. (1) comes to the conventional center of weight algorithm; when $\alpha$ is larger than 1 (such as $1.5,2,3$, etc.), Eq. (1) represents the modified versions.

\section{Error Analysis.}

The errors in the spot image may be caused by several reasons, and they are independent of each other. They generally include sample error, background noise error, photon noise error, and readout noise error[12]. The sample error is caused by the discrete sampling of the optoelectronic sensors array in the image acquisition process. It consists of two aspects: one is on the discrete intensity sampling values; the other is on the discrete spatial sampling grids. Accordingly, the sample error increases while the power factor $\alpha$ increases or the profile size of the spot decreases. The errors of background noise, photon noise and readout noise are usually mixed, and reflects in the spot image as random errors. In this paper, we call them the noise error.

Figure 1 shows a laser spot position detection experiment. Figures 1(a) and 1(b) are two frames selected from a bundle of sequential field experimental spot images. The former with laser spots is called the active image while the latter without spots is called the passive image. By subtracting Fig.1(b) from Fig.1(a), the difference image is generated and shown in Fig.1(c). It has been enhanced by intensity stretching for better presentation. Fig.1(d) displays a zoomed region of interest with laser spots of Fig.1(a), and Fig.1(e) is the 3D illustration of this region of interest. As subfigure 1(e) shows, the noise is not negligible compared to the spot signal. In this case, the noise error elimination is difficult. Because the edge of the spot is intertwined with random noise, and the center of the spot does not suffice to make the random noise negligible by measuring its intensity or some power of its intensity. Proper threshold selection for the conventional threshold method becomes very hard and sensitive to the precision of spot centroid detection. Therefore, the spot edge determination become a key problem to be solved.
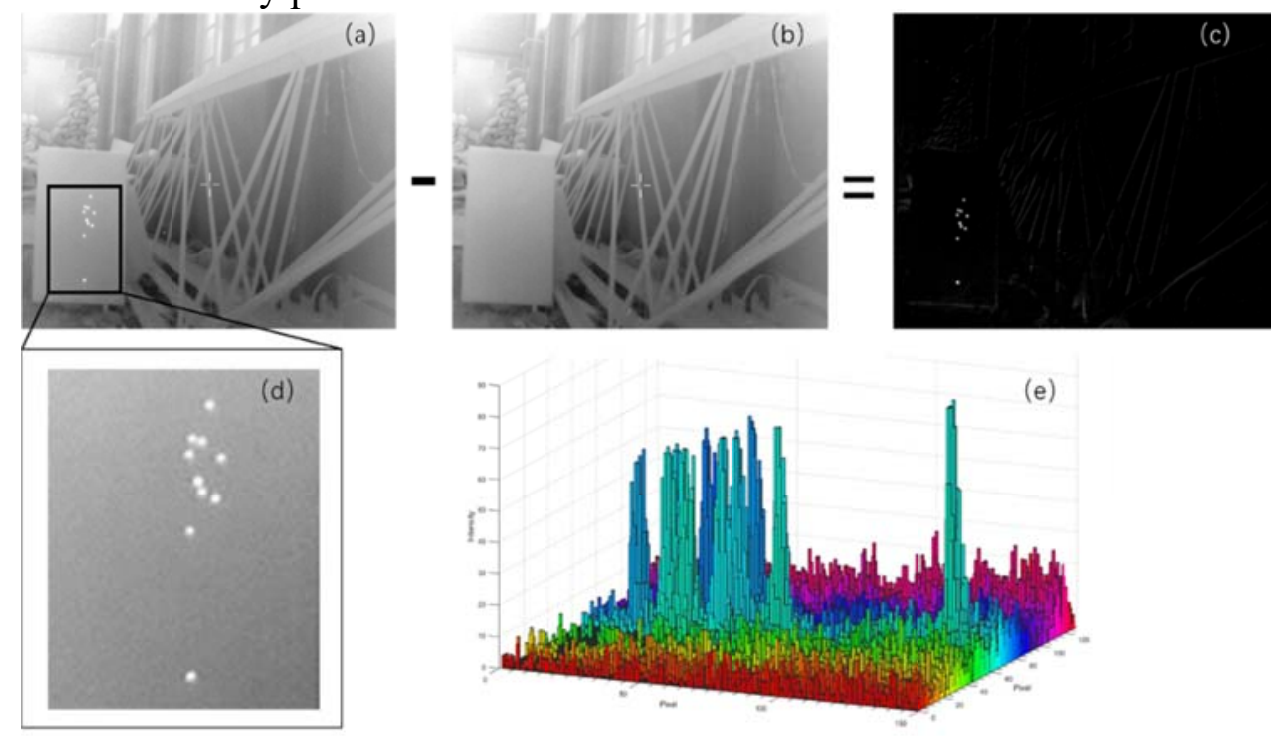

Fig. 1 Illustration of laser spot experiment. (a) one frame in sequential images with laser spot on the target board, (b) another frame without laser spot. (c) the difference image of (a) and (b), and the intensity has been stretched for a better visual effect. (d) the zoomed region of the laser spots and (e) the $3 \mathrm{D}$ illustration of this region. 


\section{Proposed Algorithm.}

\section{1) Noise Error Estimation}

The spot edge determination has two steps: first the random noise errors should be estimated and roughly eliminated by a calculated threshold; second the proposed algorithm is utilized to meticulously determine the edge. For the purpose of noise error estimation, a statistic of difference images between every two sequential frames is obtained before the occurrence of a spot. The non-zero pixels contain all the information of sample error and noise error, and we can obtain the max value of the errors through the statistic. Then the max value of the errors is selected to be a threshold for eliminating noise errors, i.e. the pixels with a value less than the threshold is identified as noise error and its value is set to be 0 . The rough position of a spot is identified by using the threshold, however, some pixel of the spot on its edge is also eliminated for its value may be equal or smaller than the threshold. Therefore, the second step of spot edge determination is needed to fix the erroneously operated pixels.

\section{2) Morphological Filters}

Denoted a morphological image as set $\boldsymbol{B}$. Then took an open operation on $\boldsymbol{B}$ with a structure operator $w$. The open operation, denoted as $B \circ w$, is expressed as

$$
B \circ w=(B \odot w) \oplus w,
$$

where $B \ominus w$ represented the erosion operation, and $B \oplus w$ represented the dilation operation[13].

\section{3) Spot Edge Determination}

The spot position can be roughly located by searching the difference image with the threshold. Then we extract the spot region by a $16 \times 16$ detection window, as shown in Fig. 2(a). The spot is accompanied with random noise. A calculated threshold of 11 is taken to eliminate the random noise and extract the spot. The generated morphological image labelling the spot region is as shown in subfigure (b). Taken the morphological image (b) as a mask, we obtain the roughly detected spot region as shown in subfigure (c). As the subfigure shows, the edge of the spot profile is difficult to precisely determine by the threshold, for some pixels of the noise are falsely retained while some of the spot edge are falsely discarded. To solve this problem, morphological filters are taken on Fig. 2(b) to further eliminate the isolated noise pixels and fix the edge pixels of the spot. Subfigure (d) shows the profile of the finally extracted spot. Connective regions have been outlined in subfigures (b), (c), and (d) for better visualization.
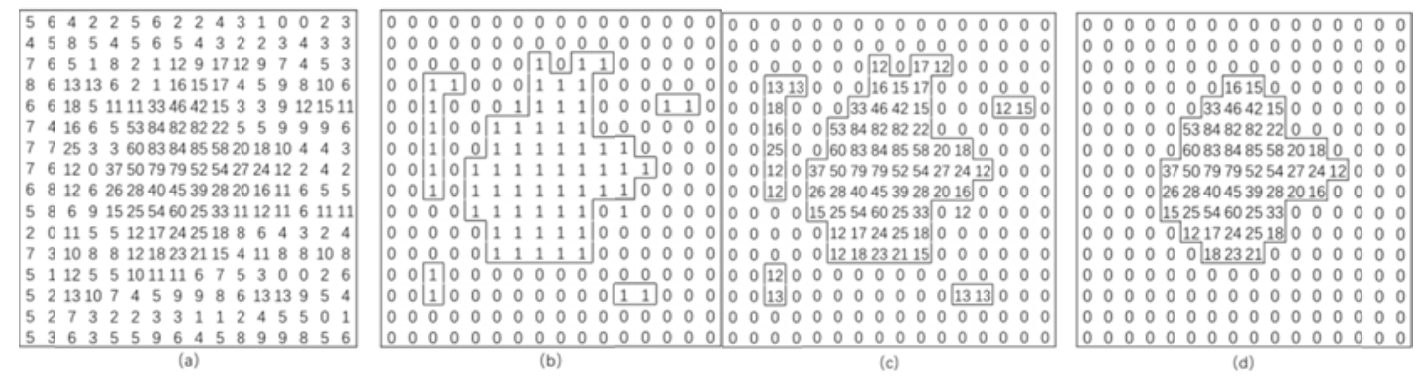

Fig. 2 The spot edge determination: (a) is the gray levels of the spot region in the difference image within the $16 \times 16$ detection window; (b) is the morphological image of the spot region after thresholding with a threshold of 11 ; (c) is the spot region after noise elimination; and (d) is the finally extracted spot.

Denote the image spot region (Fig. 2(a)) as S. Its morphological image filtered with the calculated threshold $\eta$ is denoted as $\mathbf{S}_{b w}$ and has been shown in Fig. 2(b). $\mathbf{S}_{b w}$ is produced from $\mathbf{S}$ with a Heaviside function which is defined as 


$$
\mathbf{S}_{b w}(i, j)=\left\{\begin{array}{l}
1, \mathbf{S}(i, j)>\eta \\
0, \mathbf{S}(i, j) \leq \eta
\end{array},\right.
$$

where $\mathbf{S}_{b w}(i, j)$ and $\mathbf{S}(i, j)$ are the intensity of the pixel located in position $(i, j)$ in each of them. The morphological image $\mathbf{S}_{b w}$ is then taken open operation with a structure operator $\omega$, and produces the filtered image $\mathbf{S}_{b w}^{\prime}$.

$$
\mathbf{S}_{b w}^{\prime}=\mathbf{S}_{b w} \circ \omega=\left(\mathbf{S}_{b w} \ominus \omega\right) \oplus \omega
$$

where the structure operator $\omega$ selects a diamond profile with radius of 1 , which is as

$$
\omega=\left[\begin{array}{lll}
0 & 1 & 0 \\
1 & 1 & 1 \\
0 & 1 & 0
\end{array}\right]
$$

$\mathbf{S}_{b w}^{\prime}$ is regarded as index image and indicates the precisely extracted spot profile. The filtered spot region image $\mathbf{S}^{\prime}$ is generated by the index $\mathbf{S}_{b w}^{\prime}$ with another Heaviside function which is defined as

$$
\mathbf{S}^{\prime}(i, j)=\left\{\begin{array}{ll}
\mathbf{S}(i, j), & \mathbf{S}_{b w}^{\prime}(i, j)=1 \\
0 \quad, \mathbf{S}_{b w}^{\prime}(i, j)=0
\end{array} .\right.
$$

$\mathbf{S}^{\prime}$ has been shown in Fig. 2(d).

\section{Field Experiments and Discussions}

The field experiments were performed in the laser testing range. The receiving camera was set fixed directing to the target board and captured the sequential video images. The laser emitter shot every 4 5 seconds. The laser spot shot on the target board lasted for more than 40 seconds and then began to disappear. We capture the multiple frames right after the occurrence of the spot on the target board for repeated tests. The frame sampling frequency of the commercial camera is usually between 24 to 30 frames/s, resulting in that the capture time cost of one frame is 0.03 to 0.04 second. Since the acquisition duration was much shorter than the time of a laser spot lasted until it began to disappear. We considered that the differences between every acquired sequential images were mainly due to sample error and noise error rather than the fading effect of the laser spot itself. Because the true positions of the spot centroids cannot be obtained, we defined an assessment criterion for evaluating the precision of the centroid detection.

\section{Assessment Criterion.}

Take the procedure of locating the centroid position of spot as one detection test. The detected position of spot $\xi$ on the $\zeta$-th test is denoted as $\left(x_{c}^{\xi}, \mathrm{y}_{c}^{\xi}\right)_{\zeta}$, where $\xi=1 \cdots K, K$ is the number of laser spots on the target board; $\zeta=1 \cdots N, N$ is the number of total tests. The assessment criterion is defined as

$$
M_{\xi}=\frac{1}{N} \sum_{\zeta=1}^{N}\left\|\left(x_{c}^{\xi}, \mathrm{y}_{c}^{\xi}\right)_{\zeta}-\left(\bar{x}_{c}^{\xi}, \overline{\mathrm{y}}_{c}^{\xi}\right)_{\zeta}\right\|_{2},
$$




$$
\bar{M}=\frac{1}{K} \sum_{\xi=1}^{K} M_{\xi}
$$

Where $M_{\xi}$ represents the evaluation of the centroid detection result for spot $\xi \cdot\left(\bar{x}_{c}^{\xi}, \overline{\mathrm{y}}_{c}^{\xi}\right)_{\zeta}$ is the statistical mean value of the detected centroid position in different tests for the same spot $\xi . \bar{M}$ is the statistical mean value of the measurements criterion for all tests of the entire spots. A smaller value of the criterion indicates a better precision of the results.

Experimental Results and Analysis.

To validate the repeatability, stability and precision of our algorithm, we first calculated the centroid position for one single spot with multiple tests. Figure 3 shows four of them for the same laser spot. Subfigures $\left(S_{1}\right)-\left(S_{4}\right)$ were the extracted spot region by a threshold of 11 in different tests. $\left(S_{1}^{\prime}\right)-\left(S_{4}^{\prime}\right)$ shows the extracted spot region by our algorithm.

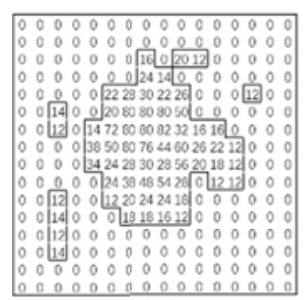

$\left(S_{1}\right)$

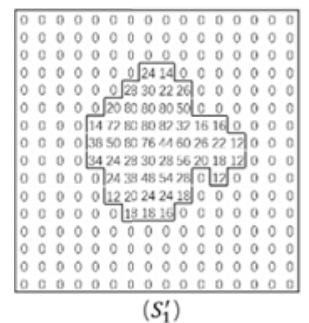

$\left(S_{1}^{\prime}\right)$

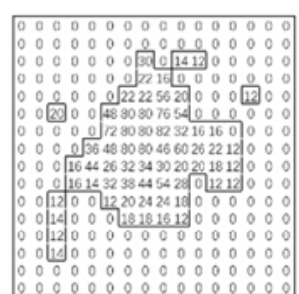

$\left(S_{2}\right)$

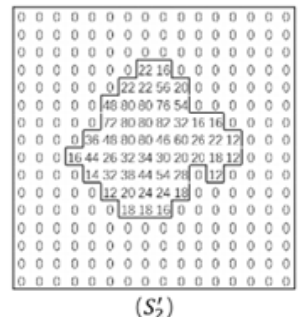

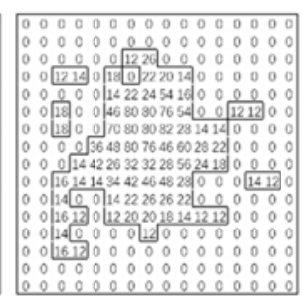

$\left(S_{3}\right)$

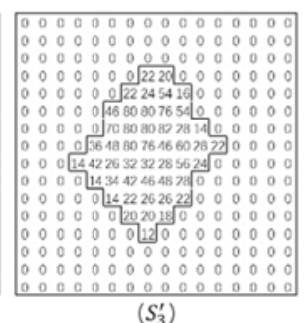

$\left(S_{3}^{\prime}\right)$

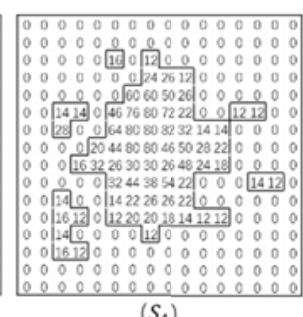

$\left(S_{4}\right)$

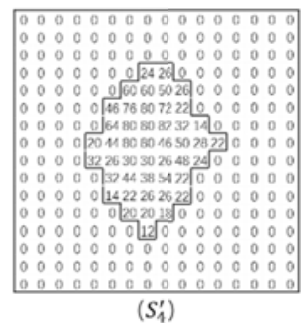

Fig. 3 The comparison of the threshold method and our proposed algorithm. $\left(S_{1}\right)-\left(S_{4}\right)$ are the extracted spot profiles by using the conventional threshold method in four different tests for the same spot. $\left(S_{1}^{\prime}\right)-\left(S_{4}^{\prime}\right)$ are the detected spot profiles by using our proposed algorithm.

As shown in Fig. 3, the profiles of detected spots in the first row were irregular and some pixels of noise were still retained. The second row showed more regular and compact spot regions than the first row, and no obvious noise pixels were retained. Then we took 20 tests for a statistical result. The centroid is calculated by Eq. (1) with the power of 1, i.e. the conventional center of weight algorithm. The detected spot centroids by the proposed algorithm and the reference method are shown in Fig. 4.

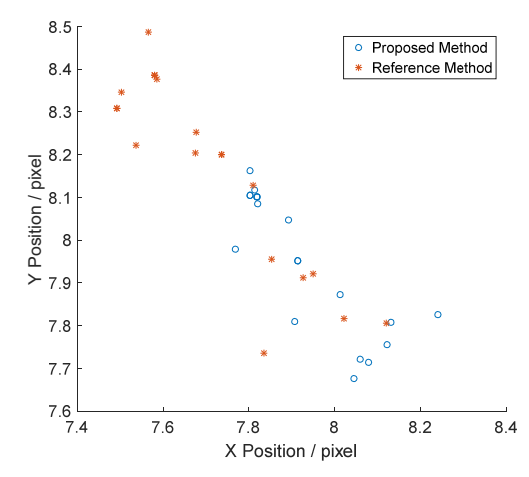

Fig. 4 The distribution of the detected spot centroids in the repeated tests by the proposed method and reference method. 
As shown in Fig.4, the centroid distribution of our method is more compact than that of the reference method. It demonstrates that our method has superior stability for centroid positioning. The detected centroid positions with their confidence intervals $(\alpha=0.05)$ and assessment criterion are shown in Table 1.

Table 1 The statistical result of centroid detection for one certain spot.

\begin{tabular}{ccc}
\hline Method & Centroid $(\boldsymbol{\alpha}=\mathbf{0 . 0 5})$ & $\boldsymbol{M}_{\xi}$ \\
\hline Proposed Method & $(7.9296 \pm 0.0613,7.9495 \pm 0.0711)$ & 0.1727 \\
Reference Method & $(7.7134 \pm 0.0825,8.1669 \pm 0.0997)$ & 0.5043 \\
\hline
\end{tabular}

As shown in Table 1, both of the confidence intervals and the assessment criterion value demonstrate the superiority of the proposed method on centroid location precision. Considering the influence of the threshold selection, we recalculated the centroid with the threshold variance from 11 to 20. For each threshold, we also took 20 tests for statistic. The distribution of detected centroids with threshold variance is shown in Fig. 5.

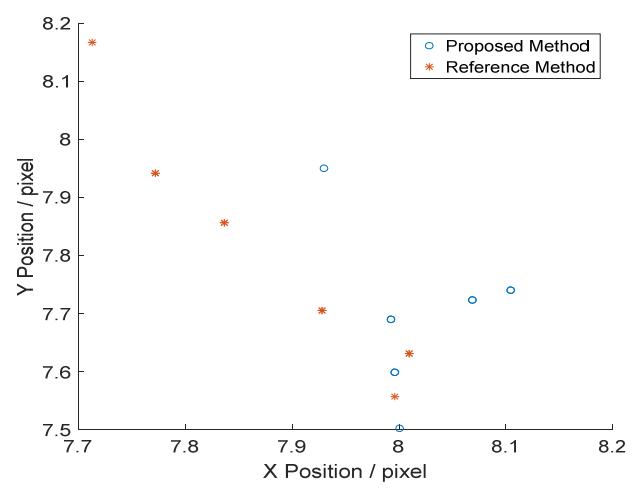

Fig. 5 Distribution of the detected spot centroids with threshold variance from 11 to 20.

In Fig. 5, the proposed method also shows a more compact distribution than the reference method, which demonstrates that our method has superior stability. The detailed result is as shown in Table 2.

Table 2 The result of centroid location with threshold variance for one certain spot.

\begin{tabular}{c|cc|cc}
\hline \multirow{2}{*}{ Threshold } & \multicolumn{2}{|c|}{ Proposed Method } & & Reference Method \\
\cline { 2 - 6 } & Centroid $(\mathbf{\alpha}=\mathbf{0 . 0 5})$ & $\boldsymbol{M}_{\boldsymbol{\xi}}$ & Centroid $(\mathbf{\alpha}=\mathbf{0 . 0 5})$ & $\boldsymbol{M}_{\boldsymbol{\xi}}$ \\
\hline 11 & $(7.9296 \pm 0.0613,7.9495 \pm 0.0771)$ & 0.1727 & $(7.7134 \pm 0.0825,8.1669 \pm 0.0997)$ & 0.5043 \\
12 & $(8.1044 \pm 0.0196,7.7405 \pm 0.0217)$ & 0.2183 & $(7.7723 \pm 0.0508,7.9424 \pm 0.0571)$ & 0.3671 \\
13 & $(8.1044 \pm 0.0196,7.7405 \pm 0.0217)$ & 0.2183 & $(7.7723 \pm 0.0508,7.9424 \pm 0.0571)$ & 0.3671 \\
14 & $(8.0691 \pm 0.0167,7.7239 \pm 0.0201)$ & 0.1368 & $(7.8365 \pm 0.0531,7.8558 \pm 0.0493)$ & 0.3475 \\
15 & $(8.0691 \pm 0.0167,7.7239 \pm 0.0201)$ & 0.1368 & $(7.8365 \pm 0.0531,7.8558 \pm 0.0493)$ & 0.3475 \\
16 & $(7.9929 \pm 0.0158,7.6901 \pm 0.0219)$ & 0.2330 & $(7.9276 \pm 0.0312,7.7052 \pm 0.0364)$ & 0.3050 \\
17 & $(7.9929 \pm 0.0158,7.6901 \pm 0.0219)$ & 0.2330 & $(7.9276 \pm 0.0312,7.7052 \pm 0.0364)$ & 0.3050 \\
18 & $(7.9957 \pm 0.0221,7.5983 \pm 0.0218)$ & 0.3835 & $(8.0092 \pm 0.0284,7.63113 \pm 0.0238)$ & 0.4005 \\
19 & $(7.9957 \pm 0.0221,7.5983 \pm 0.0218)$ & 0.3835 & $(8.0092 \pm 0.0284,7.63113 \pm 0.0238)$ & 0.4005 \\
20 & $(8.0009 \pm 0.0363,7.5025 \pm 0.0189)$ & 0.4482 & $(7.9960 \pm 0.0365,7.5564 \pm 0.0464)$ & 0.5064 \\
\hline
\end{tabular}

As shown in Table 2, the confidence intervals and assessment criterion values of the proposed method are smaller than those of the reference method, which also indicates a validation of the superior stability of the former method.

For further validation of repeatability, we calculated the centroid positions for different laser spots. The laser spots shot on the target board and their detected centroid positions by both of the proposed and reference methods are shown in Fig. 6. 

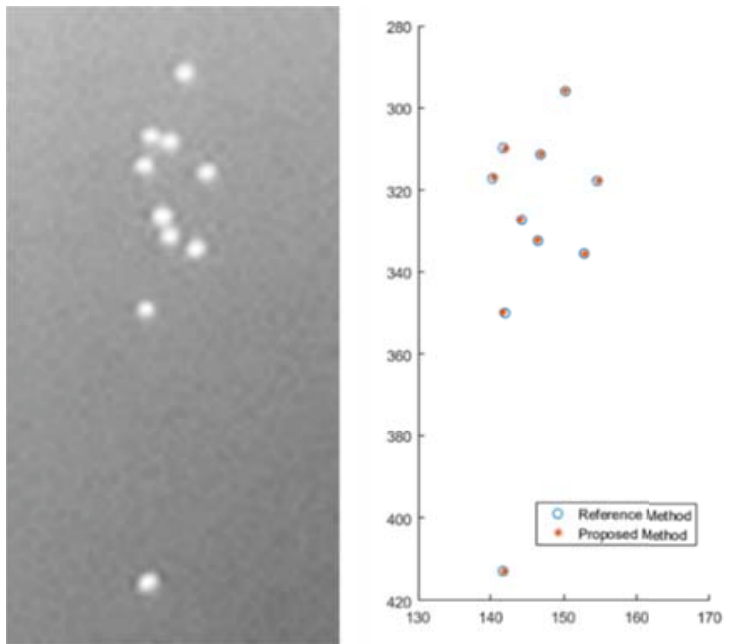

Fig. 6 The experimental result of centroid location for multiple spots.

As shown in Fig. 6, there were totally 10 spots which had been tested. The calculated centroid positions and the evaluations are listed in Table 3.

Table 3 The result of centroid location for the entire testing laser spots.

\begin{tabular}{|c|c|c|c|c|}
\hline \multirow{2}{*}{ Spot } & \multicolumn{2}{|l|}{ Proposed Method } & \multicolumn{2}{|l|}{ Reference Method } \\
\hline & Centroid ( $\alpha=0.05)$ & $M_{\xi}$ & Centroid ( $\alpha=0.05)$ & $M_{\xi}$ \\
\hline 1 & $(295.8147 \pm 0.0275,150.1576 \pm 0.0210)$ & 0.1971 & $(295.8926 \pm 0.0501,150.2606 \pm 0.0463)$ & 0.5785 \\
\hline 2 & $(309.9058 \pm 0.0253,141.9706 \pm 0.0372)$ & 0.2708 & $(309.6736 \pm 0.0329,141.7365 \pm 0.0448)$ & 0.4858 \\
\hline 3 & $(311.1270 \pm 0.0406,146.9572 \pm 0.0362)$ & 0.3859 & $(311.3016 \pm 0.0893,146.8456 \pm 0.0778)$ & 0.6960 \\
\hline 4 & $(316.9134 \pm 0.0418,140.4854 \pm 0.0165)$ & 0.3930 & $(317.1304 \pm 0.0743,140.2585 \pm 0.0567)$ & 0.6691 \\
\hline 5 & $(317.6324 \pm 0.0175,154.8003 \pm 0.0148)$ & 0.2014 & $(317.7217 \pm 0.0575,154.5988 \pm 0.0426)$ & 0.5512 \\
\hline 6 & $(327.0975 \pm 0.0296,144.1419 \pm 0.0299)$ & 0.2974 & $(327.2264 \pm 0.0584,144.3036 \pm 0.0499)$ & 0.5670 \\
\hline 7 & $(332.2785 \pm 0.0278,146.4218 \pm 0.0484)$ & 0.2807 & $(332.4001 \pm 0.0594,146.5192 \pm 0.0747)$ & 0.6165 \\
\hline 8 & $(335.5469 \pm 0.0359,152.9157 \pm 0.0236)$ & 0.4050 & $(335.4930 \pm 0.0985,152.8243 \pm 0.0979)$ & 0.7342 \\
\hline 9 & $(349.9575 \pm 0.0384,141.7922 \pm 0.0334)$ & 0.3728 & $(350.0341 \pm 0.0429,142.0173 \pm 0.0663)$ & 0.5357 \\
\hline 10 & $(412.9296 \pm 0.0613,141.9495 \pm 0.0771)$ & 0.1727 & $(412.7134 \pm 0.0825,142.1669 \pm 0.0997)$ & 0.5043 \\
\hline $\bar{M}$ & 0.2977 & & 0.5938 & \\
\hline
\end{tabular}

As shown in Table 3, the centroid confidence intervals and the performance assessment criterion values of each spot have demonstrated the superiority of our proposed method. The statistical mean value of the measurements criterion for our method is 0.2977 . It is smaller than that of the reference method which is 0.5938 . This result also indicates the superiority of the presented method over the reference one.

\section{Conclusion}

This study proposed a novel centroid location method based on profile regularization. The method improves the precision of the center detection algorithm by accurately determining the edge of the spot profile and accurately eliminating random noise errors. It doesn't need an estimation of the optimal threshold for eliminating background and random noise. In this way, it avoids the decrease in precision due to the threshold selection. Additionally, this method can be taken as a previous process step of the modified center of weight algorithms. The experimental results have demonstrated the superiorities of our proposed method over the reference method on precision, stability and repeatability. The proposed method has shown its great value in spot centroid detection which may contribute to various research areas. 


\section{References}

[1] Z. Jiang, S. Gong, and Y. Dai, "Numerical study of centroid detection accuracy for Shack-Hartmann wavefront sensor," Optics \& Laser Technology, vol. 38, 614-619, 2006.

[2] M. Nicolle, T. Fusco, G. Rousset, and V. Michau, "Improvement of Shack-Hartmann wave-front sensor measurement for extreme adaptive optics," OPTICS LETTERS, vol. 29, no. 23, pp. 2743-2745, 2004.

[3] J. E. Greivenkamp, D. G. Smith, R. O. Gappinger, and G. A. Williby, "Optical Testing Using Shack-Hartmann Wavefront Sensors,” Proceedings of SPIE, vol. 4416, pp. 260-263, 2001.

[4] J. Schwiegerling, and E. DeHoog, "Problems testing diffractive intraocular lenses with Shack-Hartmann sensors," Applied Optics, vol. 49, no. 16, pp. D62-D68, 2010/06/01, 2010.

[5] H. Chen, and C. Rao, "Accuracy analysis on centroid estimation algorithm limited by photon noise for point object," Optics Communications, vol. 282, pp. 5, 2009.

[6] W. Guo, L. Zhao, C. S. Tong, C. I-Ming, and S. C. Joshi, "Adaptive centroid-finding algorithm for freeform surface measurements,” APPLIED OPTICS, vol. 52, no. 10, pp. D75-D83, 2013.

[7] D. W. Kang, M. Kang, and J. W. Hahn, "Measuring two-dimensional profiles of beam spots in a high-density spot array for a maskless lithography system," APPLIED OPTICS, vol. 53, no. 36, pp. 8507-8513, 2014.

[8] X. Yu, D. Zhao, and L. Chen, "Adaptation of adaptive optics system.," Proceedings of SPIE, vol. 3126, pp. 432, 1997.

[9] S.-H. Baik, S.-K. Park, C.-J. Kim, Y.-S. Seo, and Y.-J. Kang, "A new centroid algorithm for shack-hartmann wavefront sensor," Proceedings of SPIE, vol. 4926, pp. 251-260, 2002.

[10]S.-K. Park, and S.-H. Baik, "A study on a fast measuring technique of wavefront using a Shack-Hartmann sensor," Optics \& Laser Technology, vol. 34, no 8, pp. 687-694, 2002.

[11] J. F. Ren, C. H. Rao, and Q. M. Li, "An Adaptive Threshold Selection Method for Hartmann-Shack Wavefront Sensor,” Opto-electronic Engineering, vol. 29, no. 1, 1-5, 2002.

[12]H. Li, H. Song, C. Rao, and X. Rao, "Accuracy analysis of centroid calculated by a modified center detection algorithm for Shack-Hartmann wavefront sensor," Optics Communications, vol. 281, pp. 750-755, 2008.

[13]R. C. Gonzalez, and R. E. Woods, Digital image processing, p.^pp. 520-525, Beijing:

Publishing House of Electronics Industry, 2007. 